\title{
Improved Use of Wind Turbine Kinetic Energy for System Frequency Support
}

\author{
Guoyi $\mathrm{Xu}^{1 *}$, Lie $\mathrm{Xu}^{2}$ \\ ${ }^{1}$ State Key Laboratory of Alternate Electrical Power System with Renewable Energy Sources, \\ North China Electric Power University, Beijing, China \\ ${ }^{2}$ Department of Electronic and Electrical Engineering, University of Strathclyde, Glasgow, \\ U.K. \\ *xu_gy@ncepu.edu.cn
}

\begin{abstract}
To ensure security operation of power systems with high wind penetration, wind turbines (WTs) are required to participate system frequency control. The amount of WT kinetic energy used for system frequency control is discussed and minimum rotor speeds according to different WT operation states are defined to avoid large drop of mechanical power during WT frequency control. The effect of different power shapes of releasing the kinetic energy on system frequency support is investigated and two methods are proposed. The first one is aimed at reducing the rate of change of frequency (ROCOF) whereas the second one aimed to reduce both the ROCOF and frequency nadir. The proposed strategies not only make full use of the available kinetic energy but also lead to a smooth transition when the rotor re-accelerates. The performance of the proposed strategies is validated by simulations using Matlab/Simulink. The results indicate significant improvement on system frequency control.
\end{abstract}

\section{Introduction}

With high penetration of wind power in power systems, wind power plants are required to provide ancillary services to fulfil the requirements of the power system operation[1][2]. The widely installed variable speed WTs decouple the generator from the power network by converters, and do not naturally respond to system frequency change. When a frequency drop occurs the system can experience large ROCOF and large frequency nadir due to the reduced effective system inertia. Such a problem is particularly severe in isolated systems or systems with very high wind power penetration. However, the use of power electronic interface enables fast power control of the variable speed WTs which could be beneficial for system frequency control.

Various control strategies have been proposed for variable speed WTs to participate system frequency control [3]-[15]. In order to provide system frequency support, WTs need to supply extra power to the network which can come either from the deloaded WTs [3], [4] or the kinetic energy stored in the rotating mass [5]-[15]. Compared with the deloading method utilizing kinetic energy has attracted more attention as it does not influence the normal operation of the WTs and the WTs still operate at MPPT curve to have the maximum power production. Generally, there are two groups of methods to utilize the kinetic energy of variable speed WTs for system frequency control. The first group is to emulate inertial response and droop response of conventional generators by set up the artificial link between system 
frequency, ROCOF and WT output power. This method is easy to implement and has attracted many attentions [5]-[10]. However, it is difficult to select proper controller parameters to provide the right balance between the risk of WT stall and the amount of released kinetic energy for frequency support. Another method uses the kinetic energy to produce a fixed amount of excess power during frequency drop [12]-[15]. Because it is relatively easy to select the controller parameters which can be quantified according to different wind speeds, this method has been adopted by some industries [15]. However, when the WTs operate from deceleration to acceleration, the large power deficit can cause another potentially large frequency drop [12].

Recent researches have been trying to optimize the control effect while minimize the drawbacks by focusing on, estimating the available kinetic energy [16]-[18], optimizing power reserve [19] and coordinating the WT control with other energy storage sources [20], [21]. In [16], a probabilistic approach is used to estimate the aggregate inertial response available from WT. By tuning the tip speed ratio, reference [17] proposed a strategy to estimate and control the kinetic energy for the WT to provide frequency regulation. In order to have high kinetic energy storage with minimum loss of power production, reference [18] formulated an optimal method to achieve a minimum loss of power production and minimum inertial reserve requirement. In [19], differential evolution methods were used to optimise the rotational speed and the pitch angle to maximise the rotational kinetic energy. These methods aim to optimize the control effect and maximise the kinetic energy by combined pitch control and rotor speed control. Reference [20] proposed a coordinated control methods of WT and superconducting magnetic energy storage device to avoid the second frequency drop. A fuzzy logic method was adopted in [21] to manage the wind-storage system for frequency control using again kinetic energy.

The effect on system frequency control depends on the amount of available kinetic energy and the power shape of energy release. This paper presents improved control strategies for using WT's kinetic energy for system frequency control. The control strategies aim to fully use the available kinetic energy to support ROCOF and frequency nadir, whereas in the same time minimize the side effect during WT rotor re-acceleration. The paper is organized as follows. WT system modelling is presented in Section 2 and power system frequency control is briefly discussed in Section 3. The minimum rotor speed and available kinetic energy for system frequency control is investigated in Section 4. Two power shapes to release the kinetic energy from WTs for system frequency control are studied in Section 5. Simulation results are presented in Section 6 and finally, Section 7 draws conclusions. 


\section{WT system modelling}

There are generally two types of variable speed WTs, doubly fed induction generator (DFIG) and fully rated converter based WTs. Due to their superior control ability, the fully rated converter based WTs have attracted many attentions. Various generator topologies have been used including induction generators, permanent magnet synchronous generators (PMSG) and electrically excited synchronous generators. This paper focuses on the fully rated converter WT using PMSG though the proposed control principle is also applicable to other variable speed WTs. The WT system includes WT model and its control.

WTs extract power from wind and convert it to mechanical power which then drives the rotor of the wind generator. The mechanical power extracted from wind can be expressed as [22]

$$
\begin{gathered}
P_{m}=\frac{1}{2} \rho A V_{w}^{3} c_{p}(\lambda, \beta) \\
c_{p}(\lambda, \beta)=c_{1}\left(\frac{c_{2}}{\lambda_{i}}-c_{3} \beta-c_{4}\right) e^{\frac{-c_{5}}{\lambda_{i}}}+c_{6} \lambda \\
\frac{1}{\lambda_{i}}=\frac{1}{\lambda+0.08 \beta}-\frac{0.035}{\beta^{3}+1}
\end{gathered}
$$

where $\rho$ is air density, $A$ is turbine swept area, $V_{w}$ is wind speed, $\beta$ is blade pitch angle, the coefficients are $c_{1}=0.5176, c_{2}=116, c_{3}=0.4, c_{4}=5, c_{5}=21$ and $c_{6}=0.0068, \lambda$ is tip speed ratio of the rotor blade tip speed to wind speed.

During operation maximum power point tracking (MPPT) is usually implemented and is given as

$$
P_{\text {opt }}=k_{\text {opt }} \omega_{r}^{3}
$$

where $\omega_{r}$ is the rotor speed, $k_{\text {opt }}$ is the coefficient of the MPPT curve and is given as

$$
k_{\text {opt }}=0.5 \rho \pi R_{b}^{5} C_{\text {pmax }} / \lambda_{\text {opt }}^{3}
$$

where $R_{b}$ is the radius of the blades, $C_{\text {pmax }}$ is the maximum performance coefficient, $\lambda_{\text {opt }}$ is the optimal tip ratio. All these parameters are dependent on the design of the WT. In this paper, the WT used for analysis has a rated wind speed of $12 \mathrm{~m} / \mathrm{s}$. Under the rated wind speed, the output maximum power is $0.7 \mathrm{pu}$ and the turbine operates at the maximum rotor speed of $1.2 \mathrm{pu}$. Below rated wind speed, the WT is operated at MPPT. When the maximum turbine speed of $1.2 \mathrm{pu}$ is reached, the control strategy changes to control the turbine speed at $1.2 \mathrm{pu}$ and increases output power with the increasing wind speed. Once the rated WT 
output power is reached (wind speed larger than $13.8 \mathrm{~m} / \mathrm{s}$ ), pitch control is activated to maintain constant output power.

The control of the WT consists of two parts, i.e. turbine control and converter control. The turbine control includes speed control and power control which generate the pitch angel and power reference for the converter. The WT converter contains the generator side converter (GSC) and the line side converter (LSC). The GSC is directly connected to the PMSG for controlling its operation. The LSC controls the DC link voltage and reactive power output to the AC network. As the control and operation of such systems are well understood [22], no more description is given here.

\section{System frequency control}

The frequency drop in power system can be described by many indices, the ROCOF $d \Delta f(t) / d t$, frequency nadir $\Delta f_{\max }$, steady state frequency deviation $\Delta f_{n}$, time to reach frequency nadir $T_{\text {nadir }}$. For these indices, the ROCOF and frequency nadir are of most concern, as many under frequency load shedding techniques use these indices for making decisions [23].

Under normal control variable speed WTs do not provide inertial response, thus no contribution to system inertia. The equivalent inertia of the whole system $H_{s y s}$ can be represented as

$$
H_{\text {sys }}=\frac{\sum_{i=1}^{n}\left(\frac{1}{2} J_{i} \omega_{m}^{2}\right)}{S_{s y s}}
$$

where $n$ is the number of conventional generators, $J$ is the combined moment of inertia of the conventional generators and turbines, $\omega_{m}$ is the rated mechanical angular velocity of the rotor, $S_{s y s}$ is the rating of the whole power system including the wind generation. It is observed from (6) that high penetration of wind power in power system will reduce equivalent system inertia.

In load frequency control, the relationship between frequency deviation and power imbalance can be expressed as

$$
2 H_{\text {sys }} \frac{d \Delta f}{d t}+D \Delta f=P_{\text {gen }}+P_{\text {wind }}-P_{\text {load }}
$$

where $D$ is load damping constant, $P_{\text {gen }}$ represents generation from conventional generators. It will cause large ROCOF and large frequency nadir especially when the equivalent system inertia is low due to the large penetration of wind power in the power system. However, due to fast power control ability of WTs, frequency support could be provided by WTs through auxiliary control. From (7) it can be seen that at the beginning of a frequency event, $\Delta f$ is relatively small, and thus the ROCOF will be largely dependent on 
the power imbalance and $H_{\text {sys }}$. If the WT is controlled to produce more power in this period, the power imbalance will be reduced and so as the ROCOF. It needs to be noted that the detection of frequency variation and the subsequent activating frequency support can cause initial delay in providing frequency response but such delay is likely to be fairly short, e.g. hundreds of milliseconds range.

\section{Wind turbine kinetic energy}

The kinetic energy of a WT consists of the kinetic energy of the rotating blades, the gearbox and the generator. For the direct driven WT using PMSG, due to the large mass and size of the rotor, the WT has a high inertia, which can be even larger than the equivalent synchronous generators [9].

The kinetic energy released from a machine with a rotor speed change of $\Delta \omega_{m}$ is

$$
\Delta E=2 H S\left(\omega_{m}^{2}-\left(\omega_{m}-\Delta \omega_{m}\right)^{2}\right)
$$

where $H$ and $S$ are the inertial constant and power rating of the WT. In this study, a 3 MW fully rated PMSG with an inertial constant of $6 \mathrm{~s}$ is considered for analysis. The maximum kinetic energy that can be released from the WT according to (8) is $11.9 \mathrm{MJ}$ when the rotor speed change from $1.2 \mathrm{pu}$ to $0.7 \mathrm{pu}$.

WT rotor speed reduces when the WT is controlled to release kinetic energy for frequency support. In the meantime, the captured mechanical power also drops as the WT is no longer operate at the maximum power point. The mechanical power reduction rate with rotor speed change $\left(d P_{m} / d w_{r}\right)$ can be obtained by differentiating the mechanical power given in (1), and the results for different wind speeds are shown in Fig. 1. For wind speed higher than $13.8 \mathrm{~m} / \mathrm{s}$, pitch controller is activated to regulate the output power the WT, and the pitch angle can be reduced to increase the captured mechanical power of the WT during frequency support in addition to the utilization of the kinetic energy (limited by the overload capabilities of the WT and converter), which is not considered in this paper.




Fig. 1.WT mechanical power reduction rate with rotor speed change $\left(d P_{m} / d \omega_{r}\right)$ for various wind speeds and operating rotor speeds

As can be observed in Fig. 1, in the WT operation range (rotor speed from 0.7 to $1.2 \mathrm{pu}$ ), the mechanical power captured by the WT decreases faster when the rotor speed becomes lower. For example, at wind speed of $12 \mathrm{~m} / \mathrm{s}$, the reduction rate increases from approximately $0.35 \mathrm{pu}$ at rotor speed of $1.1 \mathrm{pu}$ (point A in Fig. 1) to 1.2 pu at rotor speed of 0.8 pu (point B in Fig. 1). In addition, the reduction of the captured mechanical power with rotor speed change is higher for higher wind speed. If the WT is controlled for system frequency support, although it can be operated to the minimum speed $0.7 \mathrm{pu}$, due to the low mechanical power captured at such a low rotor speed, the total output power from WT will be less. Thus, there is limited benefit to operate at such speed range during frequency support. Taking the wind speed of $12 \mathrm{~m} / \mathrm{s}$ as an example, the turbine initially operates at 1.2 pu speed in normal state and produces $0.7 \mathrm{pu}$ output power. If the rotor speed decreases to $0.7 \mathrm{pu}$ while the wind speed remains at $12 \mathrm{~m} / \mathrm{s}$, the output power will drop to $0.33 \mathrm{pu}$, which is less than half of the normal output power. The power reduction is more significant if the wind speed is higher than $12 \mathrm{~m} / \mathrm{s}$. Based on above observations, the minimum rotor speeds during frequency support for different wind speeds are shown in Fig. 2(a) and set as follows:

- For wind speed less than $11 \mathrm{~m} / \mathrm{s}$, the mechanical power reduction rate is relatively lower (less than 1.0 as shown in Fig. 1), and in order to have adequate WT kinetic energy for system frequency support, the minimum allowed rotor speed is chosen to be $0.7 \mathrm{pu}$.

- For wind speed higher than $11 \mathrm{~m} / \mathrm{s}$ but less than rated wind speed $12 \mathrm{~m} / \mathrm{s}$, the minimum rotor speed is chosen to ensure that $d P_{m} / d \omega_{r}$ is less than 1.0 (see Fig. 1). In such cases the rotor speed will have reduced operation ranges since the minimum allowed rotor speed is higher than $0.7 \mathrm{pu}$ as shown in Fig. 2 a). The available kinetic energy for frequency control is slightly lower, but the WT will have a small reduction of mechanical power.

- For wind speed higher than $12 \mathrm{~m} / \mathrm{s}$, the rotor speed is kept at $\omega_{\max }$ (i.e. $1.2 \mathrm{pu}$ ) in normal state, and the captured mechanical power decreases very fast when the rotor speed drops as illustrated in Fig. 1. In order to limit the mechanical power drop while maintain adequate kinetic energy supply during frequency control, the minimum rotor speed is set to the same value for wind speeds above rated speed of $12 \mathrm{~m} / \mathrm{s}$ (i.e. $0.87 \mathrm{pu}$ as shown in Fig. 2 (a)). Thus the available kinetic energy which can be used for frequency support is identical for wind speed above $12 \mathrm{~m} / \mathrm{s}$. 


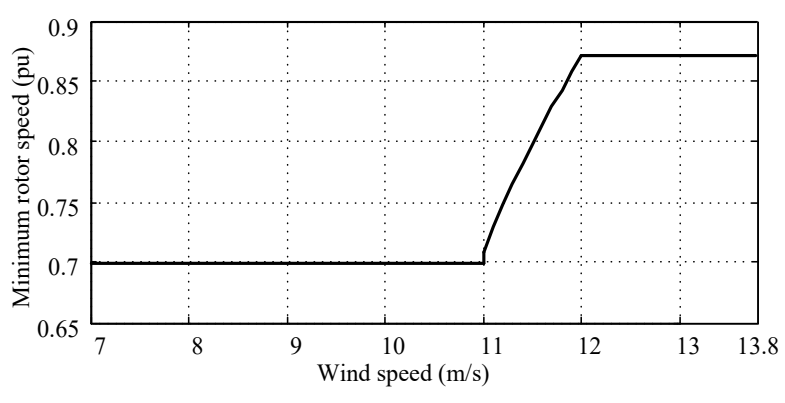

a

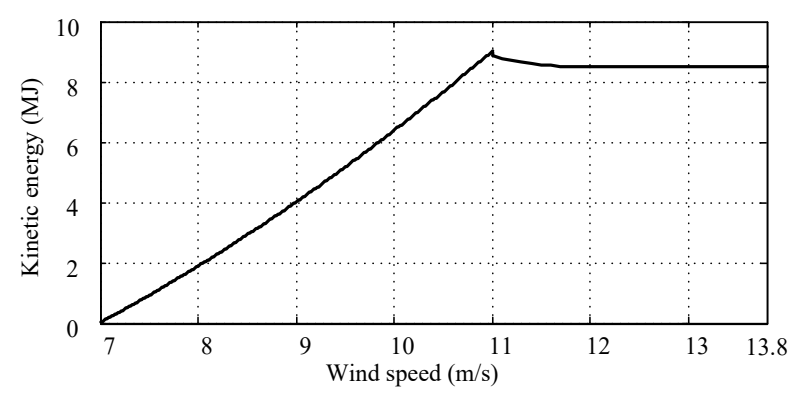

b

Fig. 2.Minimum rotor speed and available kinetic energy a Predefined WT minimum rotor speed for frequency control b Available kinetic energy of different wind speeds

With the WT's minimum rotor speed defined for frequency control, the available kinetic energy from the WT for different wind speeds can be calculated by (8) and the results are shown in Fig. 2(b). It can be observed that the WT's available kinetic energy increases with the wind speeds, for wind speeds higher than $11 \mathrm{~m} / \mathrm{s}$, because a higher minimum operation speed is chosen to avoid large mechanical power drop, the available kinetic energy reduces slightly. It needs to be noted that during higher wind speed, the captured mechanical power by the wind turbine is increased resulting in higher overall power from the turbine (i.e. the sum of the captured mechanical power and additional power from inertial response).

\section{Frequency control of variable speed WTs}

\subsection{WT frequency control strategy}

The controller for WT system frequency control using kinetic energy is shown in Fig. 3(a). It consists of mechanical power estimator \& MPPT, frequency event detector and frequency controller. The generated power reference signal $P_{\text {ref }}$ is sent to the GSC controller to control the power generation of PMSG.

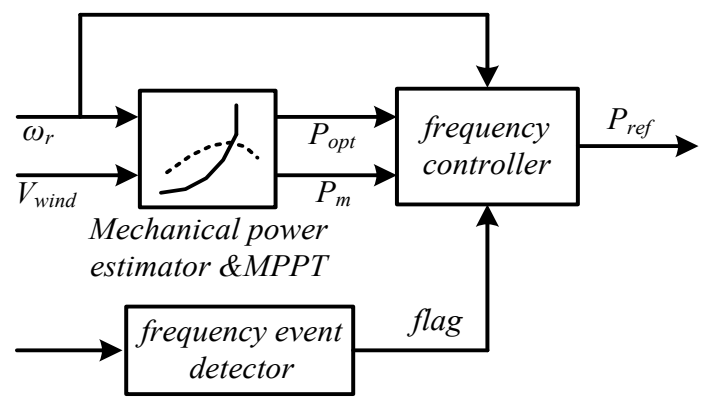





Fig. 3. Block diagram of WT's active power controller

a Diagram of WT power controller

b WT MPPT controller

c Frequency drop detector

\subsubsection{Mechanical power estimator \& MPPT:}

The wind speed is measured and the mechanical power is obtained through an estimator according to (1). The control for MPPT is illustrated in Fig. 3(b), where the reference value of the electrical output power $P_{\text {opt }}$ is determined by the rotor speed. A PI controller with limited output is used to limit the rotor speed to be less than the maximum rotor speed.

\subsubsection{Frequency event detector:}

A frequency relay is used to detect abnormal power system frequency. The system frequency signal is first passing through a high pass filter to eliminate the steady state error. A hysteresis controller is then adopted to detect the abnormal frequency as shown in Fig. 3(c). If the system frequency drop is larger than the threshold $f_{m}$, the flag is set to 1 to activate the WT frequency control. The value of $f_{m}$ can be set to provide adequate sensitivity but avoid activating WT's frequency control during minor frequency variations. When the system frequency variation returns to the value $f_{n}$, the flag is set to 0 to end frequency support.

\subsubsection{Power control strategy:}

When abnormal frequency is detected, the WT is controlled to provide system frequency support by releasing kinetic energy. During this process, the power reference $P_{r e f}$ is set to be greater than the captured mechanical power $P_{m}$, and the power imbalance will decelerate the rotor. However, due to the converter capacity limit, the maximum output power of the WT is limited to 1.2 pu rated power, i.e. a $20 \%$ overload capability for the converter for a duration of up to 1 minute is assumed in this study. The captured 
mechanical power will decrease with the decrease of rotor speed, as discussed in Section 4. When the rotor speed reaches the pre-set minimum speed the rotor stops decelerating. The deceleration time $T_{d e c}$ from the initial state to the minimum speed is affected by the output power setting.

The power reference for the rotor deceleration period is of most concern as it determines the frequency support effect. At the beginning of a frequency deviation, due to the fast power control ability of variable speed WTs, they can be controlled to produce higher power at the beginning of the frequency drop so as to reduce the ROCOF. When the rotor speed approaching the minimum value, $P_{\text {ref }}$ can be set to be close to $P_{m}$ to reduce the power deficit when the frequency support process ends and the rotor accelerates. Thus a function related to the rotor speed is proposed to produce the output power reference as

$$
P_{\text {ref }}=P_{m}+K \times\left(\frac{\omega_{r}-\omega_{\min }}{\omega_{r 0}-\omega_{\min }}\right)
$$

where $\omega_{\min }$ is the predefined minimum rotor speed. $\omega_{r}$, and $\omega_{r 0}$ are the actual and initial rotor speeds when the frequency drop occurs, respectively. $K$ is a gain which defines the deceleration time $T_{d e c}$, and the calculation of $K$ will be discussed in the next section.

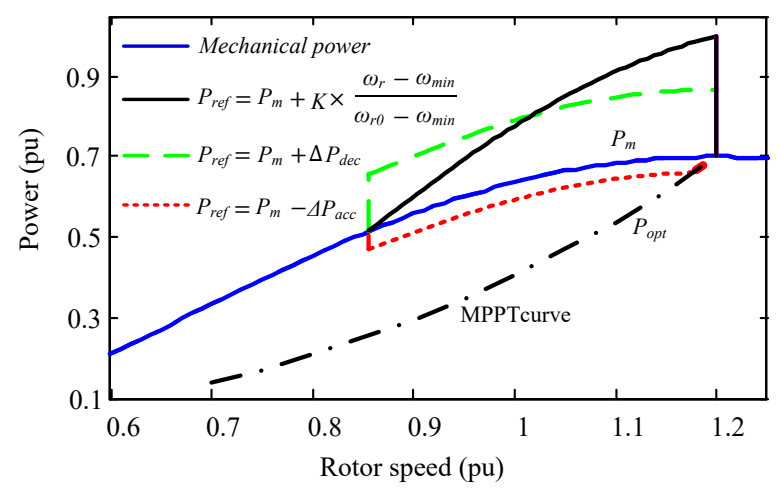

Fig. 4.Power curve of $W T$

For the proposed power setting, the power imbalance will be

$$
\Delta P=K \times\left(\frac{\omega_{r}-\omega_{\min }}{\omega_{r 0}-\omega_{\min }}\right)
$$

The power reference is illustrated in Fig. 4. As can be observed that the WT generates the highest power at the beginning of the frequency deviation, and then $\Delta P$ decreases with the reduction of rotor speed and equal to zero when the rotor speed reaches the predefined minimum value. In this case, the WT will have a significant contribution to the reduction of ROCOF and a smooth transition will also be achieved to 
avoid the second frequency drop. This strategy also takes consideration of the pre-frequency drop operation states to fully use the available kinetic energy.

Another control strategy considered is to have a constant power imbalance during the deceleration period as

$$
P_{\text {ref }}=P_{m}+\Delta P_{\text {dec }}
$$

According to Section 4, after defining the minimum rotor speed and $T_{d e c}$, with the available kinetic energy from (8), the parameter $\Delta P_{d e c}$ can be calculated. The power reference is illustrated in Fig. 4. Compared with the previous control strategy for ROCOF support with the same $T_{d e c}$, it can be observed in Fig. 4 that at the beginning of the frequency drop, the second method has a lower output power resulting in slower rotor speed reduction. However, in the duration of frequency drop (rotor speed less than $1.0 \mathrm{pu}$ in Fig. 4), it has a higher output power so as to reduce frequency nadir. However when the rotor changes to acceleration, the power deficit will be $\Delta P_{d e c}+\Delta P_{a c c}$, which could cause another frequency drop, though it is likely to be much smaller than the method with fixed power [12].

When the rotor speed reaches the predefined low limit, the operation point needs to return back to the MPPT curve. In order to accelerate the WT, the power reference $P_{\text {ref }}$ is set to be lower than $P_{m}$ to produce an acceleration torque, bringing the rotor back to the optimum speed. There are many strategies to control the active power reference during the re-acceleration stage and in this paper a simple method adopted is to set the $P_{r e f}$ as

$$
P_{\text {ref }}=P_{m}-\Delta P_{a c c}
$$

where $\Delta P_{a c c}$ is a constant which can be chosen to be relatively small to avoid a large power step when transiting from deceleration (frequency support) to acceleration. The active power output increases with the increase of rotor speed as shown in Fig. 4. The WT will eventually move to the MPPT curve and operates at the optimal speed, as shown by the acceleration curve in Fig. 4.

\subsection{Parameter calculation}

To implement the two frequency control strategies, the parameter $K, \Delta P_{d e c}$ in (9) and (11) need to be determined. The block diagram based on equation (13) for calculating the relationships between the controller parameters $K, \Delta P_{d e c}$ and $T_{d e c}$, is illustrated in Fig. 5(a). The maximum power reference for the 
WT is limited to $1.2 \mathrm{pu}$ as previously described. For different values of $K$ and $\Delta P_{\text {dec }}$, the duration of the WT to reach minimum allowed rotor speed can be obtained from the simulation, and the results of the relationship between $K, \Delta P_{d e c}$ and $T_{d e c}$ are plotted in Figs. 5 (b) and (c). For implementation, the results for different wind speeds can be stored in a look-up table, and once $T_{d e c}$ is selected the controller parameter values can be fetched from the table. It is observed from Figs. 5 (b) and (c) that larger $T_{d e c}$ will result in a smaller $K$ and $\Delta P_{d e c}$. In Fig. 5 (b), for high wind speed (such as $13 \mathrm{~m} / \mathrm{s}$ ), the captured mechanical power is high, the total output power of the WT (mechanical power and power from kinetic energy) will hit the 1.2 rated power limit, resulting in a increased $T_{d e c}$ (i.e. longer frequency support duration) for the same value of $K$. In Fig. 5 (c), as the available kinetic energy for wind speed of $11 \mathrm{~m} / \mathrm{s}$ is slightly higher than that of wind speed above $12 \mathrm{~m} / \mathrm{s}$ as shown in Fig. 2 (b), for the same $T_{d e c}$ a slightly larger additional power $\left(\Delta P_{d e c}\right)$ can be obtained for $11 \mathrm{~m} / \mathrm{s}$ compared to that of $12 \mathrm{~m} / \mathrm{s}$ or higher for the same $T_{d e c}$.

$$
2 H \frac{d \omega_{r}}{d t} \omega_{r}=\Delta P=P_{m}-P_{r e f}
$$


b 


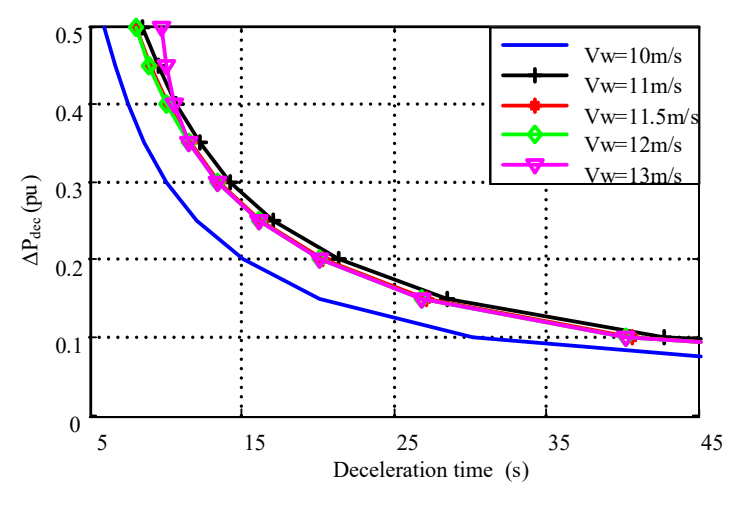

Fig. 5.Block diagram of calculation relationship of controller parameter and $T_{\text {dec }}$ and results a Block diagram of calculate control parameters

$\mathrm{b}$ Relationship of $\mathrm{K}$ and $\mathrm{T}_{\mathrm{dec}}$ under different wind speeds

c Relationship of $\Delta \mathrm{P}_{\mathrm{dec}}$ and $\mathrm{T}_{\mathrm{dec}}$ under different wind speeds

\section{Simulations}

To evaluate the proposed control strategies, simulation analyses have been carried out using Matlab/Simulink. A power system model as illustrated in Fig. 6 is developed and the system has a total load of $3600 \mathrm{MW}$ and a $500 \mathrm{MW}$ wind farm represented by a lumped fully rated converter based WT. There are two synchronous generators, G1 and G2 rated at 2000 MW and 3000 MW respectively to represent the main AC networks. The two generators are modelled with an IEEE type 1 automatic voltage regulator and a reheat steam turbine. The model of the governor and turbines and their parameter values are shown in the appendix. The speed governor is modelled with a proportional (droop) and integral frequency control loop. The frequency droop of the governor is $4 \%$ and the integral control loop recovers the system frequency to nominal value after frequency event to emulate the secondary frequency control. The system frequency is measured by a voltage PLL at the wind farm connecting point $\mathrm{A}$.

In the case study, the wind speed is $11.4 \mathrm{~m} / \mathrm{s}$ and the WT operates at MPPT mode. The rotor speed is $1.14 \mathrm{pu}$ and the WT produces $300 \mathrm{MW}$ active power. The wind speed is assumed to be constant during the simulation. 


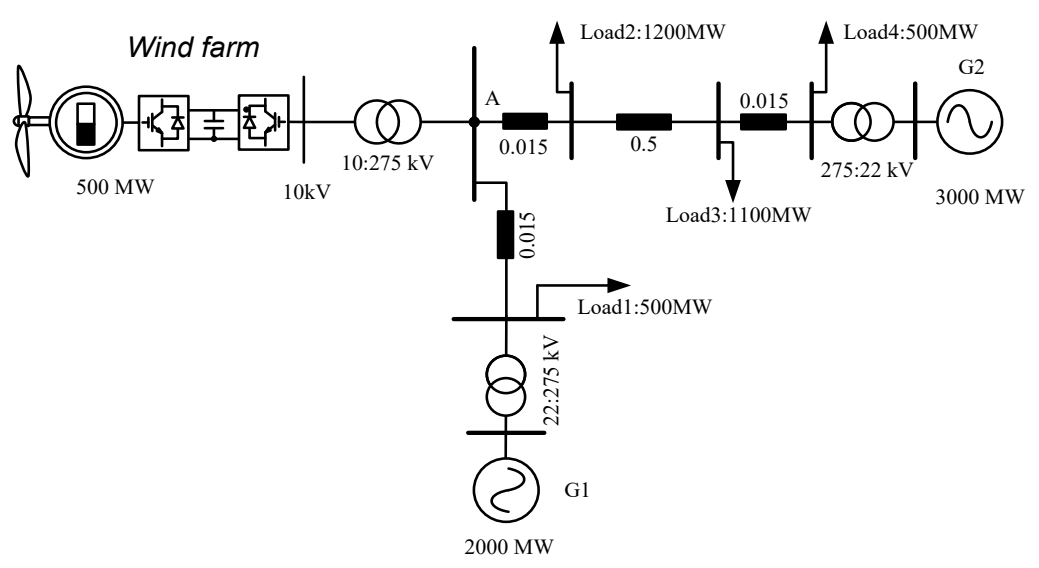

Fig. 6.Simulated power system

For the wind speed of $11.4 \mathrm{~m} / \mathrm{s}$, the predefined minimum rotor speed for frequency control is 0.782 pu according to Section 4. $T_{d e c}$ is selected to be $30 \mathrm{~s}$ and the corresponded value of $K$ and $\Delta P_{d e c}$ in (9) and (11) are 0.5 and 0.145 . In order to compare the effect on frequency support with different parameter values, the cases with $T_{d e c}=60 \mathrm{~s}, K=0.21$, and $\Delta P_{d e c}=0.069$ are also simulated. For the speed recover stage, $\Delta P_{a c c}=0.08$ is assumed in the simulation cases. A sudden increase of Load1 by $300 \mathrm{MW}$ at $5 \mathrm{~s}$ is considered in the simulations to cause the system frequency drop. At about $5.5 \mathrm{~s}$ the frequency relay detects the frequency drop, and the WT frequency control is activated. The simulation results of the two control strategies are shown in Fig. 7 and Fig. 8 respectively.

Fig. 7 shows the simulation results when the WT is controlled to support ROCOF with the additional WT power varied according to the rotor speed as defined in (9). It is observed from Fig. 7(a) that the ROCOF has been significantly reduced by the WT's contribution, and the frequency drops much slower than that without WT frequency control. At the transition point when the rotor re-accelerates, it has a very small power deficit, and thus, there is no obvious frequency drop. From the WT's output power shown in Fig. 7(b) it can be seen that at the beginning of the frequency control, the WT has a very large output power to compensate the power imbalance. The power then reduces with the reduction of the rotor speed and when it reaches the minimum rotor speed of $0.782 \mathrm{pu}$ (see Fig. 7(c)), the output power drops to be slightly less than the captured mechanical power to re-accelerate the rotor. When rotor speed reaches 1.1 pu, the WT's output power becomes less than the optimal power, and thus, the WT's operation will move to the MPPT curve and slightly increase the output power, and finally settle at the rotor speed of $1.14 \mathrm{pu}$. The output power of synchronous generators G1 shown in Fig. 7(d) indicates that their responses have been delayed by the fast frequency control of the WT due to the reduced frequency variation. The 
synchronous generator G2 has similar responses as of G1, but due to space limitation the results are not shown here.

For larger $T_{d e c}(60 \mathrm{~s})$, the parameter $K$ is smaller which means the output power is less than previous case (i.e. 30s) at the beginning of the frequency drop as observed in Fig. 7(b). Consequently the rotor speed reduction is also reduced resulting in a higher output power in the during of frequency drop, which lead to a slightly higher ROCOF but lower frequency nadir as shown in Fig. 7(a).

Fig. 8 shows the results of WT controlled for reducing both ROCOF and frequency nadir where the additional power produced by the WT remains constant as defined in (11). It is observed from Fig. 8(a) that both the ROCOF and frequency nadir have been reduced. However, there is a second frequency drop when the rotor changes from deceleration to acceleration. The output power from the wind farm to the AC network as shown in Fig. 8(b) increases at the beginning of the frequency drop though it reduces with the reduction of the rotor speed. At the transition to accelerate the WT, there is a step reduction of $100 \mathrm{MW}$ which causes the second frequency drop as shown in Fig. 8(a). The WT rotor speed drops from 1.14 pu to $0.782 \mathrm{pu}$ after releasing the kinetic energy as illustrated in Fig. 8(c), again when the WT's operation change to MPPT curve (rotor speed around $1.1 \mathrm{pu}$ ) the output power increases slightly.

Compare with the results in Figs. 7(b) and (c), it is observed that, it produces less output power at the beginning of the frequency drop so the kinetic energy is released slower than the first case leading to a slower drop of the rotor speed. Therefore, when the frequency reaches the minimum level, the output power from the WT is relatively higher compared to the case with the first method leading to a reduced frequency nadir. The output power of synchronous generator G1 illustrated in Fig. 8(d) indicates similar effect as previous case with slower response than system without WT frequency control. The response of generator G2 has similar response as of G1, again due to space limitation it is not shown here. Higher $T_{d e c}$ (60s) leads to a smaller $\Delta P_{d e c}$ and smaller output power as illustrated in Fig. 8(b). This results in slower rotor speed drop but slightly higher frequency nadir as observed in Figs. 8(c) and (a). 


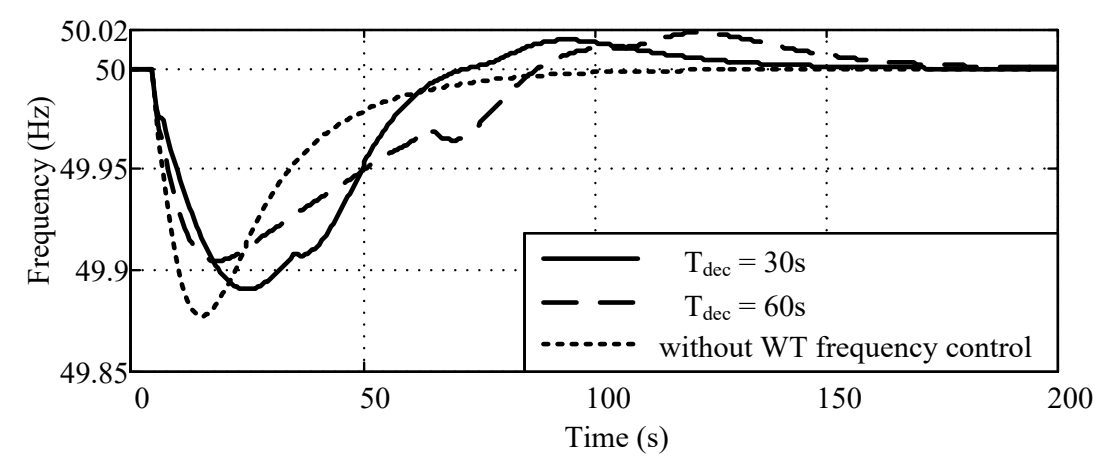

a



b
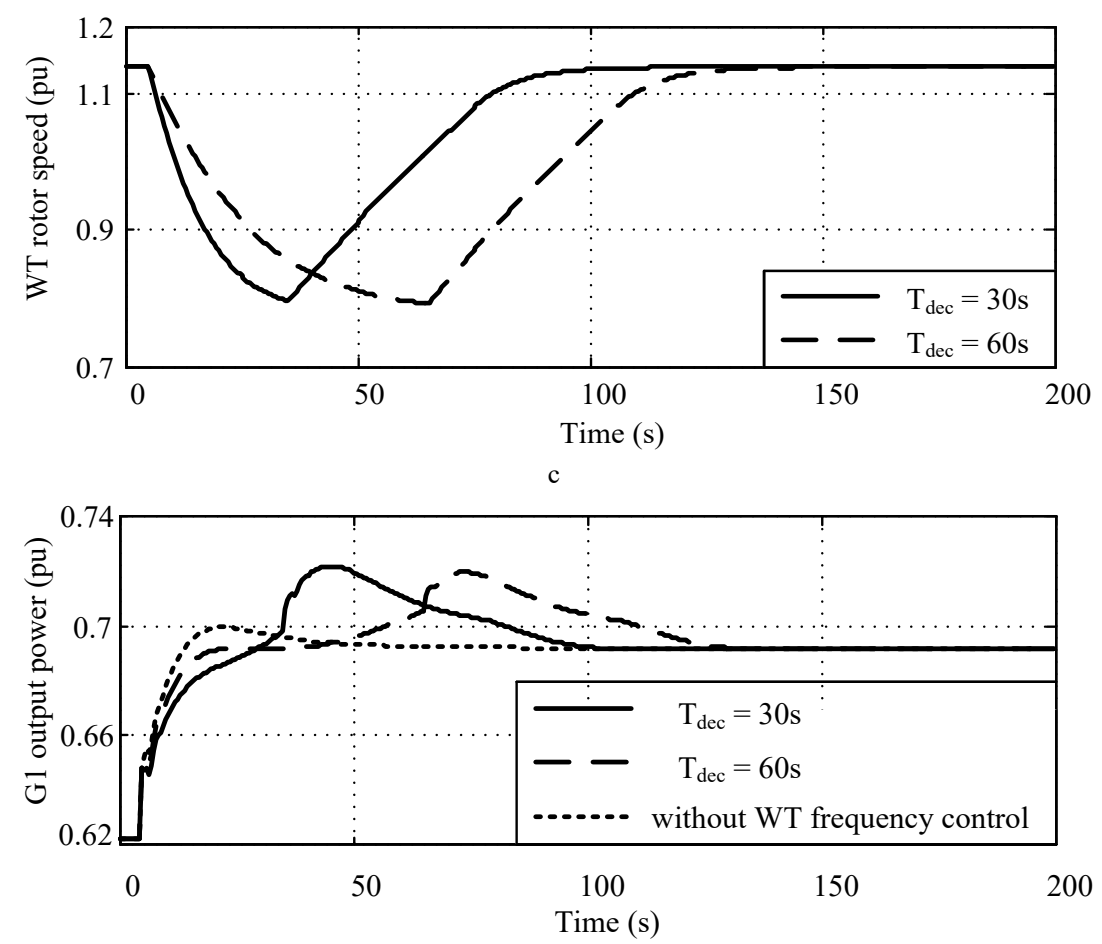

$\mathrm{d}$

Fig. 7.Simulation result with the additional power produced by the WT varying according to the rotor speed a System frequency

b Wind farm output power

c WT rotor speed

d Output power of conventional generator G1 




a

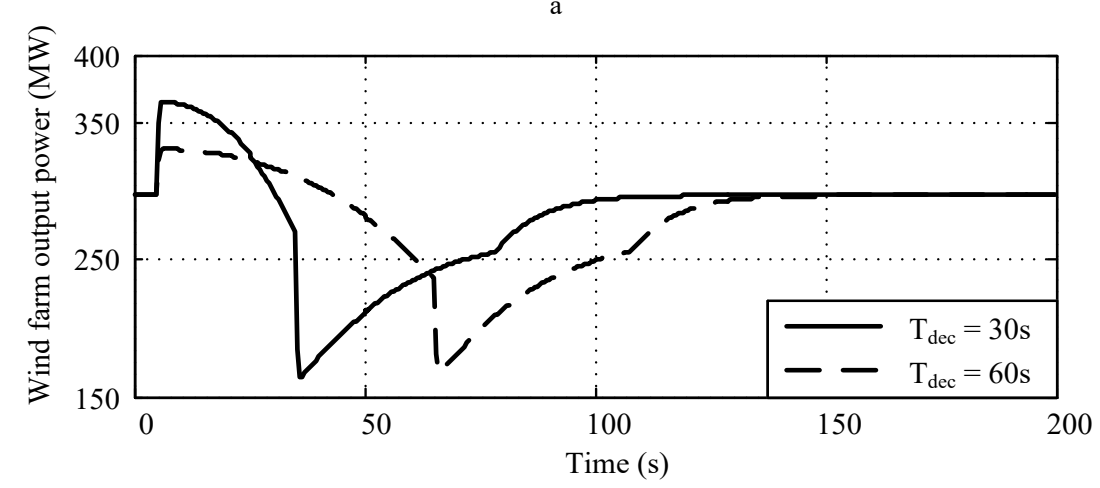

b

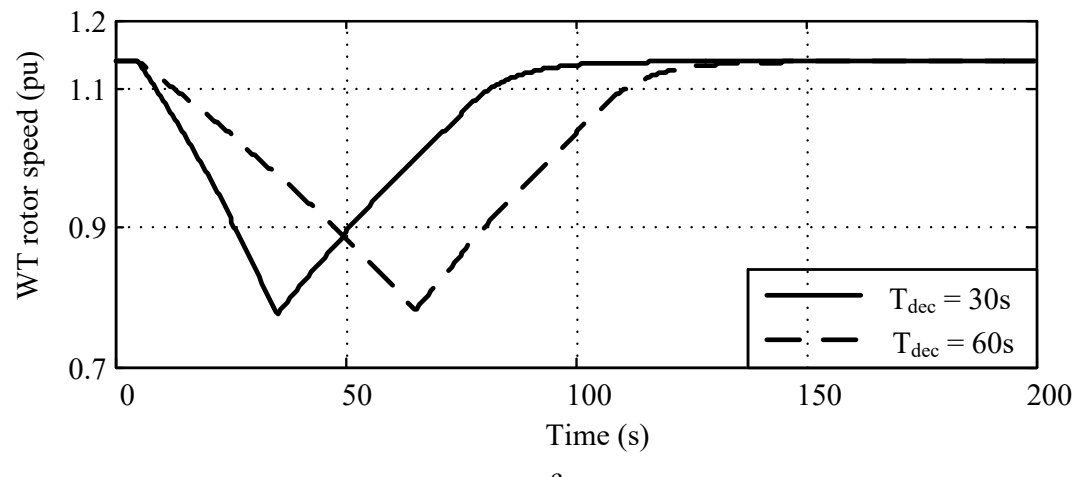

$\mathrm{c}$

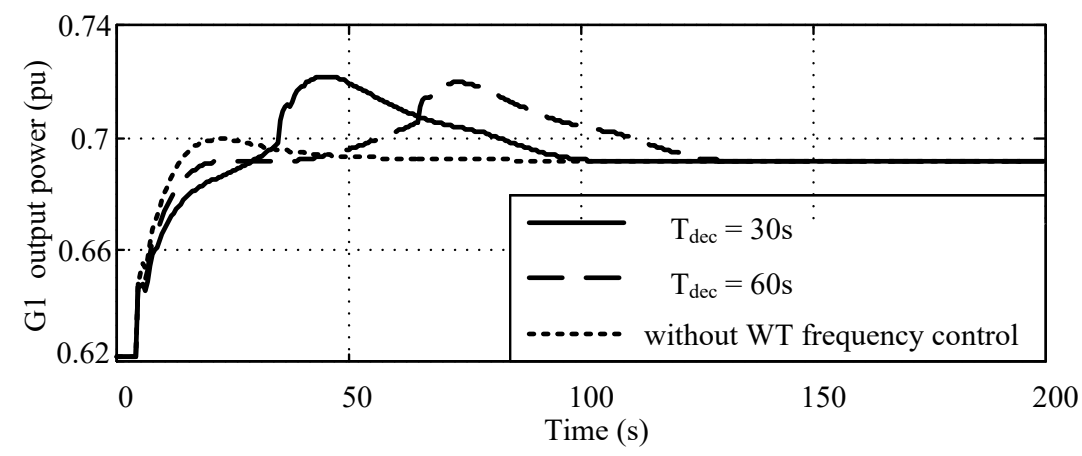

$\mathrm{d}$

Fig. 8. Simulation result with constant additional power produced by the WT

a System frequency

b Wind farm output power

c WT rotor speed

d Output power of conventional generator G1 


\section{Conclusion}

This paper has investigated the control of variable speed WTs for system frequency support. The amount of kinetic energy used for frequency control was studied and the minimum WT rotor speeds at different wind conditions were defined to avoid large drop of mechanical power during frequency support process. To make the full use of the available kinetic energy, two different power shapes were proposed with one mainly aimed for reducing ROCOF and the other improving both ROCOF and frequency nadir. The simulation results proved that the proposed scheme can significantly reduce the ROCOF and frequency nadir while in the meanwhile minimize the power imbalance when rotor is re-accelerated.

WTs have only limited and temporary kinetic energy that can be released to support the AC system, but also need to return the energy back to the WT system after frequency control. Whether to utilize the limited kinetic energy to improve ROCOF or frequency nadir has to be carefully considered and depends on the characteristics of the system. Compare with the conventional generators, the most important feature of variable speed WTs is the fast power control ability which enables WTs to regulate their power faster than the conventional generators. Thus, it might be beneficial to control the WTs to improve the ROCOF, while the conventional generators are controlled to reduce frequency nadir by coordination with the WTs.

\section{Acknowledgments}

This work was sponsored by the National Natural Science Foundation of China project under Grant 51507062.

\section{References}

[1] Tsili, M., Papathanassiou, S.: 'A review of grid code technical requirements for wind farms', IET Renew. Power Gener., 2009, 3, (3), pp. 308-332.

[2] Miller, N. W., Shao, M., Clark, K.: 'Emergency response: U.S. system frequency with high wind penetration', IEEE Power Energy Mag., 2013, 11, (6), pp. 63-71.

[3] Zhang, Z. S., Sun, Y. Z., Lin, J. et al.: 'Coordinated frequency regulation by doubly fed induction generator based wind power plants', IET Renew. Power Gener., 2012, 6, (1), pp. 38-47.

[4] Vidyanandan, K. V., Senroy, N.: 'Primary frequency regulation by deloaded wind turbines using variable droop', IEEE Trans. Power Syst., 2013, 28, (2), pp. 837-846.

[5] Margaris, D., Papathanassiou, S. A., Hatziargyriou, N. D., et al.: 'Frequency control in autonomous power systems with high wind power penetration', IEEE Trans. Sustainable Energy, 2012, 3, (2), pp. 189-199. 
[6] Lalor, G., Mullane, A., O’Malley, M.: 'Frequency control and wind turbine technologies', IEEE Trans. Power Syst., 2005, 20, (4), pp. 1905-1913.

[7] Hughes, F. M., Anaya-Lara, O., Jenkins, N., et al.: 'Control of DFIG-based wind generation for power network support', IEEE Trans. Power Syst., 2005, 20, (4), pp. 1958-1966.

[8] Ramtharan, G., Ekanayake, J. B., Jenkins, N.: 'Frequency support from doubly fed induction generator wind turbines', IET Renew. Power Gener., 2007, 1, (1), pp. 3-9.

[9] Morren, J., de Haan, S. W. H., Kling, W. L., et al.: 'Wind turbines emulating inertia and supporting primary frequency control', IEEE Trans. Power Syst., 2006, 21, (1), pp. 433-434.

[10] Morren, J., Pierik, J., de Haan, S. W. H.: 'Inertial response of variable speed wind turbines', Electr. Power Syst. Res., 2006, 76, (11), pp. 980-987.

[11] Conroy, J. F., Watson, R.: 'Frequency response capability of full converter wind turbine generators in comparison to conventional generation', IEEE Trans. Power Syst., 2008, 23, (2), pp. 649-656.

[12] Ullah, N. R., Thiringer, T., Karlsson, D.: 'Temporary primary frequency control support by variable speed wind turbines- potential and applications', IEEE Trans. Power Syst., 2008, 23, (2), pp. 601-612.

[13] Tarnowski, G. C., Kjær, P. C., Sørensen, P. E., et al.: 'Variable speed wind turbines capability for temporary over production', Proc. 2010 Power Eng. Soc. General Meeting, Jul. 2010, pp. 1-7.

[14] Brisebois, J., Aubut, N.: 'Wind farm inertia emulation to fulfill hydro-québec's specific need', Proc. 2011 Power Eng. Soc. General Meeting, Jul. 2011, pp. 1-7.

[15] Shao, M., Miller, N. W.: 'GE wind power control design', 2nd workshop on active power control from wind power, May 2015.

[16] Wu, L., Infield, D.: 'Towards an assessment of power system frequency support from wind plant-modeling aggregate inertial response', IEEE Trans. Power Syst., 2013, 28, (3), pp. 2283-2291.

[17] Attya, B. T., Hartkopf, T.: 'Control and quantification of kinetic energy released by wind farms during power system frequency drop', IET Renew. Power Gener., 2013, 7, (3), pp. 210-224.

[18] Diaz, G., Casielles, P. G., Viescas, C.: 'Proposal for optimizing the provision of inertial response reserve of variable-speed wind generators', IET Renew. Power Gener., 2013, 7, (3), pp. 225-234.

[19] Verbic, Z. G., Pantos, M.: 'Optimised control approach of frequency control contribution of variable speed wind turbines', IET Renew. Power Gener., 2012, 6, (1), pp. 17-23.

[20] Miao, L., Wen, J., Xie, H., et al.: 'Coordinate control strategy of wind turbine generator and energy storage equipment for frequency support', IEEE Trans. Ind. Appl., 2015, 51, (4), pp. 2732-2742.

[21] Zhang, S., Mishra, Y., Shahidehpour, M.: 'Fuzzy-logic based frequency controller for wind farms augmented with energy storage systems', IEEE Trans. Power Syst., 2015, 31, (2), pp. 1595-1603. 
[22] Chinchilla, M., Arnaltes, S., Burgos, J. C.: 'Control of permanent-magnet generators applied to variable-speed wind-energy systems connected to the grid', IEEE Trans. Energy Convers., 2006, 21, (1), pp. 130-135.

[23] Kundur, P.: 'Power system Stability and Control', New York: McGraw-Hill, 1994.

\section{Appendices}

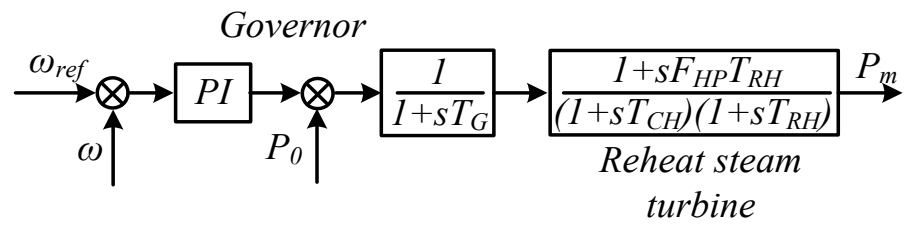

with the parameters: $T_{G}=0.2 \mathrm{~s}, T_{C H}=0.3 \mathrm{~s}, T_{R H}=6 \mathrm{~s}, F_{H P}=0.3$. 